\title{
Optimizing outcomes and treatment sequences in EGFR mutation-positive non-small-cell lung cancer: recent updates
}

\author{
Nicolas Girard*,1 \\ ${ }^{1}$ Thoracic Surgery, Institut Curie, Institut du Thorax Curie-Montsouris, Paris, France \\ *Author for correspondence: Tel.: +33 042785 7700; Fax: +33 047235 7653; nicolas.girard2@curie.fr
}

The availability of several EGFR tyrosine kinase inhibitors (TKIs) for the treatment of EGFR mutationpositive NSCLC poses important questions regarding the optimum sequence of therapy. A key consideration is how best to use the third-generation TKI, osimertinib. While osimertinib has demonstrated impressive efficacy and tolerability in a first-line setting, there are currently no standard targeted treatment options following progression. There is an argument, therefore, for reserving osimertinib for second-line use in patients who acquire the T790M resistance mutation after first- or second-generation TKIs. This article reviews recent clinical studies that have assessed the activity of sequential EGFR TKI regimens. These studies support the hypothesis that sequential use of EGFR TKIs represents a viable treatment option in 'real-world' clinical practice.

First draft submitted: 12 July 2019; Accepted for publication: 12 August 2019; Published online: 27 August 2019

Keywords: non-small-cell lung cancer • T790M • treatment sequencing

The rapid development of treatment options for patients with EGFR mutation-positive non-small-cell lung cancer (NSCLC) has proved to be one of the major models of a precision medicine approach in clinical oncology, and has demonstrated the importance of identifying targetable molecular aberrations in common tumors. Only a decade ago, the standard of care for EGFR mutation-positive NSCLC was platinum-doublet chemotherapy, and treatment outcomes were modest at best. Nowadays, clinicians have the choice of three generations of EGFR tyrosine kinase inhibitors (TKIs): the first-generation reversible EGFR inhibitors, erlotinib and gefitinib; the second-generation irreversible ErbB family blockers, afatinib and dacomitinib; and the third generation, wild-type sparing, irreversible EGFR inhibitor, osimertinib, which was originally developed to target the resistance EGFR mutation, T790M. All of these agents have demonstrated robust clinical benefit in clinical trials, with median progression-free survival (PFS) of approximately 9-18 months, and favorable tolerability profiles compared with previous standard treatment options [1-11]. As well as monotherapy, physicians also have the choice of EGFR TKI combination regimens, where appropriate. For example, randomized trials have demonstrated that the combination of erlotinib and bevacizumab is superior to erlotinib alone [12,13]. Other potential combination regimens are emerging. Recent data from the Phase III RELAY study have demonstrated superior PFS with erlotinib combined with the anti-VEGFR2 antibody, ramucirumab, versus erlotinib alone (median 19.4 vs 12.4 months; $\mathrm{p}<0.0001$ ) [14]. Also, the recent NEJ009 trial demonstrated promising outcomes in patients receiving a combination of gefitinib and platinum-doublet chemotherapy, achieving median overall survival (OS) of over 50 months [15].

With the approval of several EGFR TKIs for the treatment of EGFR mutation-positive NSCLC, the next challenge that the field faces is the identification of optimal treatment strategies for individual patients with the aim of maximizing OS. This is the most important measure of treatment efficacy in the setting of multiple lines of treatment delivered for many patients. In recent years, a number of randomized head-to-head trials have compared first- and second-/third-generation EGFR TKIs and demonstrated that afatinib, dacomitinib and osimertinib all improve PFS compared with first-generation TKIs [9-11]. As third-generation TKIs spare wild-type EGFR, they are associated with favorable tolerability compared with first-generation TKIs [11]. Second-generation TKIs, given their broad inhibitory profile, may have more frequent side effects than other TKIs, but these are generally

Future Medicine 
manageable with dose reduction and early treatment $[16,17]$. No head-to-head data currently exist that have directly compared second- and third-generation EGFR TKIs and cross-trial comparisons are not possible, not least due to differences in exclusion criteria. For example, both FLAURA (osimertinib vs gefitinib or erlotinib) [11] and LUX-Lung 7 (afatinib vs gefitinib) [9] permitted the inclusion of patients with stable brain metastases whereas ARCHER 1050 (dacomitinib vs gefitinib) did not [10]. Both osimertinib and afatinib appeared to be active against brain metastases [18-20], indicating that, unlike first-generation EGFR TKIs, they cross the blood-brain barrier at sufficient concentrations to be pharmacologically active. In FLAURA, the magnitude of osimertinib benefit was virtually identical in patients with or without brain metastases [11].

Regardless of which agent is chosen as first-line therapy for EGFR mutation-positive NSCLC, acquired resistance is inevitable. Therefore, given the broad armamentarium of treatment options, and the need for treatment sequence to achieve prolonged OS, it is important for physicians to consider possible therapy after progression [18,21,22]. A key question is whether it is best to utilize osimertinib as first-line therapy or retain it for second-line use. While osimertinib demonstrated impressive first-line activity and tolerability in FLAURA (irrespective of T790M status), no established targeted treatment options post progression have been identified yet, reflecting the heterogeneity of its resistance mechanisms [23-25]. Conversely, the predominant mechanism of resistance to first- and secondgeneration EGFR TKIs is the emergence of T790M. Osimertinib was initially approved for the treatment of T790M-positive tumors following failure of previous EGFR TKI treatment and has demonstrated striking activity in this setting [26-28]. Therefore, second line use of osimertinib remains a consideration. A key challenge of this approach is to ensure that all patients who develop T790M-driven resistance are identified. Clearly, this requires rebiopsy, and the widespread availability of sensitive T790M detection assays. However, recent developments in liquid biopsy techniques, and improvements in next-generation sequencing assays may help facilitate sequential EGFR TKI therapy in everyday clinical practice [29].

Recent findings support the use of ErbB family blockers in the first-line setting as a means to maximize OS. Exploratory analysis of ARCHER 1050 demonstrated superior OS with dacomitinib versus gefitinib (median 34.1 vs 26.8 months; hazard ratio [HR]: 0.76; 95\% CI: 0.58-0.99; $\mathrm{p}=0.044$ ) [30], which is the first time that a significant OS benefit has been shown between different EGFR TKIs. In LUX-Lung 7, afatinib demonstrated a trend toward improved OS versus gefitinib (median 27.9 vs 24.5 months; HR: 0.86; 95\% CI: 0.66-1.12; p = 0.258) [31]. Afatinib is also the only EGFR TKI to demonstrate a significant OS benefit versus platinum-doublet chemotherapy. In two Phase III trials, LUX-Lung 3 and LUX-Lung 6, afatinib significantly improved OS versus pemetrexed/cisplatin (median 33.3 vs 21.1 months; HR: 0.54; 95\% CI: 0.36-0.79; $\mathrm{p}=0.0015$ ) and gemcitabine/cisplatin (median 31.4 vs 18.4 months; HR: 0.64; 95\% CI: 0.44-0.94; $\mathrm{p}=0.023$ ), respectively, in prespecified analyses of patients with Del19 mutations [32]. This may actually reflect the delivery of sequential EGFR TKIs, as over a third of patients treated with first-line afatinib were rechallenged with another TKI [33]. Of note, in both ARCHER 1050 and the LUX-Lung studies, very few patients received subsequent osimertinib, reflecting its limited availability at the time of the trials.

Previous review articles have proposed the hypothesis that sequential use of EGFR TKIs, with osimertinib reserved for second-line use, could be a viable treatment option for many patients with EGFR mutation-positive NSCLC $[18,21,22,34]$. However, clinical data comparing sequential regimens remain scant. In this update article, very recent developments are discussed in four key areas pertinent to the feasibility of sequential regimens: emerging data on resistance mechanisms to EGFR TKIs; the latest clinical findings, from clinical trials and 'real-world' practice that have assessed the efficacy and safety of sequential EGFR TKI regimens; tolerability considerations with respect to sequential EGFR TKI regimens; potential treatment options for patients with T790M-independent resistance mechanisms.

\section{Mechanisms of resistance to EGFR TKIs}

The predominant mechanism of resistance to first- and second-generation EGFR TKIs is the emergence of T790M, which occurs in around $50-70 \%$ of cases $[28,29,35,36]$. This indicates that the majority of patients treated with these agents would be eligible for second-line osimertinib. Until recently, few data were available regarding resistance mechanisms to first-line osimertinib. However, further data have now become available and provide insights into the resistance mechanisms to second- and third-generation agents, which may help inform subsequent treatment decisions.

A number of 'real-world' studies corroborate the observation that T790M is a common mechanism of resistance to afatinib. For example, in a cohort analysis undertaken in Austria, 67 patients with acquired resistance to afatinib 
were assessed for T790M by utilizing droplet-digital PCR, a sensitive liquid biopsy technique [29]. The frequency of T790M in these patients was $73 \%$. Of note, $31 \%$ of patients had less than ten T790M copies per milliliter, which is considered to be below the detection threshold of several commercially available liquid biopsy assays [29]. Therefore, the relatively high frequency of T790M in this study probably reflects the sensitivity of the detection methodology and highlights the potential benefits of further development of liquid biopsy assays for identifying patients who could benefit from second-line osimertinib. As well as facilitating T790M testing in patients who refuse, or are ineligible for, tissue rebiopsy, sensitive liquid biopsies may identify tumors with small numbers of localized subclonal T790M-positive cells that could be missed by tissue biopsy. Other 'real-world' studies have identified T790M as a predominant resistance mechanism in patients treated with afatinib, with a detection rate ranging from 32 to $48 \%$ [37-39]. Few 'real-world' data exist that have assessed the frequency of T790M in patients who are resistant to dacomitinib. However, preclinical data indicate that it is likely to also be the predominant mechanism of dacomitinib resistance [40].

Resistance mechanisms to osimertinib appear to be very similar regardless of whether it is used in a first- or second-line setting. In an analysis of plasma from 91 patients treated with first-line osimertinib in FLAURA, the most common resistance mechanisms identified were $M E T$ amplification, identified in $15 \%$ of patients, and the emergence of the tertiary EGFR resistance mutation, C797S, in 7\% of patients [24]. Other mechanisms identified included HER2 amplification (2\%) and the emergence of PIK3CA (7\%), BRAF (3\%) or KRAS mutations (3\%). Notably, a putative resistance mechanism could not be identified in over $60 \%$ of the patients analyzed [24]. No osimertinib-treated patients were found to have T790M-positive disease at the time of resistance.

In 73 patients treated with second-line osimertinib in the AURA3 study, the most common resistance mechanisms, based on liquid biopsy, were also MET amplification (19\%) and emergence of C797S (15\%) [25]. Other mechanisms included cell-cycle gene alterations (12\%), HER2 amplification (5\%) and PIK3CA amplification/gene alterations (5\%). Of note, $19 \%$ of patients had more than one putative resistance mechanism. All patients with C797S retained T790M, and the two mutations appeared in cis, which would seemingly preclude the possibility of treating these patients with first-generation EGFR TKIs. Conversely, loss of T790M within the first weeks of osimertinib treatment has been associated with prolonged efficacy of osimertinib [41]. In another study, an analysis of 41 patients treated with second-line osimertinib found that $32 \%$ of patients retained T790M at the time of acquired resistance [23]. Interestingly, in this study, time to treatment discontinuation was shorter in patients who lost T790M, suggesting the emergence of pre-existing resistant clones. A number of competing resistance mechanisms were identified, including the emergence of KRAS mutations and targetable gene fusions.

Another recent study analyzed tumor biopsy samples from 71 patients with acquired resistance to osimertinib [42]. In this study, in contrast to liquid biopsy-based studies, the most common putative resistance mechanism was histological transformation, which was detected in $14 \%$ of samples overall, and $19 \%$ of samples from patients treated with first-line osimertinib. Tertiary EGFR mutations were more common in patients treated with later-line osimertinib (18\%) than first-line osimertinib (6\%) [42].

As the mechanisms of resistance to osimertinib are highly heterogeneous, there are no clear targeted therapy options following treatment failure; at the moment, most patients who go on to receive subsequent therapy are treated with chemotherapy [43]. Currently, clinical data on targeted treatment options are scarce and are largely based on case studies or small studies. For example, observations in two patients suggest that combination of crizotinib with osimertinib or erlotinib might overcome MET-mediated resistance [44]. Also, combination of osimertinib with the selective RET inhibitor, BLU-667, appeared to overcome RET-mediated resistance in another two patients [45]. Another potential treatment option in the future could be fourth-generation TKIs that target tertiary EGFR mutations like C797S [46]. Several studies, presented at ASCO 2019, reported promising preliminary activity with novel targeted agents in patients with EGFR mutation-positive NSCLC, including patients with acquired resistance to osimertinib, highlighting the need for patient enrollment in such ongoing clinical trials. JNJ-372 is a bispecific antibody that binds to both EGFR and cMet, and has been shown to block ligand binding, promote receptor degradation and trigger antibody-dependent cellular cytotoxicity in models of EGFR mutation-positive NSCLC [47]. In an ongoing, Phase I, first-in-human study (NCT02609776), JNJ-372 conferred a response rate of 28\% in 116 patients with EGFR mutation-positive NSCLC, including 10/47 (21\%) patients with acquired resistance to a third-generation TKI. Of these patients, four had C797S-positive tumors, one had cMet amplification and five did not have identifiable EGFR/cMet-dependent resistance [48]. Another ongoing Phase I trial is assessing the anti-HER3 antibody-drug conjugate, U3-1402, in patients with T790M-negative acquired resistance to erlotinib, gefitinib or afatinib, or acquired resistance to osimertinib (NCT03260491). In the 15 patients enrolled to date, 14 
had received osimertinib. In 13 evaluable patients, 12 patients experienced tumor shrinkage and two patients had confirmed partial responses [49].

Several ongoing early phase clinical trials are assessing novel combination regimens, with the aim of targeting signaling pathways implicated in osimertinib resistance. For example, the Phase II SAVANNAH trial is assessing the combination of osimertinib and savolitinib in patients with MET-mediated resistance to osimertinib (NCT03778229). Additionally, ongoing Phase I trials are assessing the combination of osimertinib with MEK, MET, VEGFR2 or BCL-2 family inhibitors (NCT02143466, NCT02789345, NCT02520778). The results of such trials are eagerly awaited with the hope that specific targeted regimens will become available for selected patients. However, at this time, there is an argument that osimertinib should be held back for second-line use given the current lack of targeted treatment options following osimertinib failure. Sequential use of second-generation EGFR TKIs, followed by osimertinib, could be the most effective way of maximizing the duration of chemotherapy-free treatment in patients who develop T790M-mediated resistance.

\section{Subsequent therapy after first-line TKIs: lessons from clinical trials}

Post-hoc analysis of clinical trials has demonstrated the feasibility of treating patients with sequential second-and third-generation EGFR TKIs. In a recent analysis, Park et al. assessed the uptake of, and outcomes following, subsequent therapies in 553 patients with EGFR mutation-positive (Del19/L858R) NSCLC who received firstline afatinib in LUX-Lung 3, 6 and 7 [33]. Notwithstanding the fact that osimertinib was not widely available when the LUX-Lung trials were undertaken, the analysis demonstrated that most patients received at least one subsequent line of systemic treatment following failure of afatinib. Out of the 553 patients, 394 (71\%), 265 (48\%) and 156 (28\%) received second-, third- and fourth-line treatment, respectively, at the discretion of the treating physician.

Given the lack of availability of osimertinib at the time of these studies, and the fact that molecular testing of tumor samples at progression/discontinuation was not mandatory or documented, it is unsurprising that the most common postprogression therapy was platinum-based doublet chemotherapy (50\% of patients), which was predominantly received as second-line treatment [33]. Thirty-three percent of patients were treated with single-agent chemotherapy, predominantly as third-line treatment. Other postprogression therapies included first-generation EGFR TKIs, either as monotherapy or in combination with chemotherapy, and afatinib rechallenge. Of note, uptake of subsequent therapy was particularly high $(>80 \%)$ in countries/regions with optimized comprehensive cancer care, such as Japan and the Far East, Western Europe and North America [33]. These observations indicate that most patients were sufficiently fit to receive subsequent therapy following failure of afatinib. Therefore, given high rates of T790M-mediated resistance following afatinib, the favorable tolerability profile of osimertinib, and improvements in, and access to, molecular testing techniques, it is reasonable to conclude that many patients presenting with EGFR mutation-positive NSCLC could potentially receive sequential afatinib and osimertinib.

Across LUX-Lung 3, 6 and 7, 37 patients received osimertinib following discontinuation of afatinib (27 in the greater than or equal to third-line setting). Although the sample size was small, and there was an enrichment for patients who received several lines of therapy, outcomes were encouraging. After a median follow-up of 4.7 years, median OS had not been reached and the 4-year survival rate was approximately $90 \%$. Median time on osimertinib was 20.2 months. In the ten patients who received consecutive afatinib and osimertinib, median PFS-2 was 53.3 months (95\% CI: 36.9-not reached) [33]. Although data are limited, outcomes in patients treated with sequential dacomitinib and osimertinib are also encouraging. In the ARCHER 1050 trial, 50 and $62 \%$ of patients treated with dacomitinib and gefitinib, respectively, received at least one subsequent systemic therapy [30]. As with afatinib, the most common post-progression therapy was chemotherapy (28 and 36\%, respectively). Again, few patients received a third-generation TKI (10 and $11 \%$, respectively), reflecting their limited availability at the time and low uptake of T790M testing. Nevertheless, OS in these patients was encouraging (median 36.7 months in the dacomitinib arm; median not reached in the gefitinib arm). Finally, a recent pooled analysis of the AURA extension and AURA2 trials $(\mathrm{n}=411)$, indicated that OS in patients who received sequential first-generation TKIs and osimertinib were promising; median OS with second- or later-line osimertinib was 26.8 months [50]. Also, post-hoc analysis of LUX-Lung 7 indicated encouraging outcomes in patients who received sequential gefitinib and osimertinib. In the 23 patients who received this sequence, median OS was 46.0 months [31].

Together, these observations suggest that a sequential approach of first-/second-generation EGFR TKIs followed by osimertinib could hold promise as a therapeutic strategy in patients with T790M-positive NSCLC, and could maximize the duration of chemotherapy-free treatment. It is possible that, for many patients, holding osimertinib back for second-line use could be more advantageous than its use in a front-line setting, given the current paucity 
of targeted treatment options following osimertinib failure. In a recent exploratory analysis of FLAURA, 59\% of patients who discontinued first-line osimertinib have, to date, received subsequent therapy, a rate that seems somewhat below what may be expected in a real-world clinical setting; $51 \%$ of patients were still on osimertinib at the time of the analysis [43]. Of the patients who received subsequent therapy, the most frequent treatment options were chemotherapy (56\%) followed by EGFR TKI-based regimens (35\%) [43]. In the gefitinib/erlotinib arm, 61\% of patients received subsequent treatment, again below expectations. Of these patients, $43 \%$ received osimertinib (crossover was permitted), 33\% received other EGFR TKI-based regimens and only $21 \%$ received chemotherapy. Time to discontinuation or death (median 23.0 vs 16.0 months) and PFS-2 (median not reached vs 20.0 months; HR: 0.58 ; 95\% CI: $0.44-0.78 ; \mathrm{p}=0.0004$ ) were higher in patients who received subsequent therapy in the osimertinib arm versus those in the gefitinib/erlotinib arm. While these findings might suggest that osimertinib preserves clinical benefit beyond first progression versus first-generation EGFR TKIs, they need to be interpreted with caution given the small proportion of patients who have received subsequent therapy to date. Furthermore, this analysis did not compare outcomes between patients who received osimertinib in first- and second-line settings. It remains unclear, therefore, how best to utilize osimertinib in terms of maximizing survival benefit.

Ultimately, further prospective data will be required to determine the optimal sequence of EGFR TKIs in patients with EGFR mutation-positive NSCLC. In this regard, final OS data from FLAURA will be informative in terms of comparing outcomes in patients who received first- and second-line osimertinib. Final OS data from the Phase III AURA-3 trial are also keenly awaited to assess survival in patients who received second-line osimertinib. Other trials are ongoing. The Phase II APPLE trial, for example, is assessing outcomes in patients treated with first-line osimertinib and patients receiving sequential first- and second-line (after gefitinib) osimertinib with a primary end point of 18-month PFS [51]. OS data from AURA3 and APPLE trials will provide valuable data in terms of the potential use of sequential first- and third-generation EGFR TKIs. Another ongoing Phase II trial is assessing outcomes in patients treated with intercalated osimertinib and afatinib given in 8-week cycles (WJOG10818L). However, no prospective trials have directly compared outcomes in patients receiving sequential first-/second- and third-generation EGFR TKIs versus patients who receive third-generation EGFR TKIs in the first line. This is an area of unmet need and will be required to provide definitive evidence of whether it is advantageous to hold osimertinib back for second-line use. Also the design of future studies should explore if there is any difference in OS between patients receiving sequential first- and third-generation TKIs and those receiving sequential secondand third-generation TKIs.

\section{Subsequent therapy after first-line TKIs: lessons from 'real-world' data}

As clinical trials often exclude patients with certain characteristics, for example, those with poor prognostic features such as active brain metastases, it is becoming increasingly recognized that 'real-world' data can play an important complementary role in determining the efficacy and safety of treatment regimens $[52,53]$. Several 'real-world' studies have demonstrated encouraging clinical outcomes in patients receiving sequential EGFR TKIs. One such analysis, the observational GioTag study, demonstrated promising activity of sequential afatinib and osimertinib in patients with T790M-positive tumors [54]. This study, undertaken across ten countries, retrospectively reviewed medical records of 204 patients with EGFR-TKI naive, EGFR mutation-positive NSCLC treated outside of clinical trials. In order to minimize the impact of selection bias, inclusion was restricted to a maximum of 15 consecutive patients from each participating center. The primary outcome was the time on treatment from the first dose of afatinib until the last dose of osimertinib or death. The study included a proportion of patients with poor-risk features, such as those with ECOG PS of $>1$ (15\% of patients enrolled) and those with brain metastases (10\%).

Encouragingly, the median time on treatment was over 2 years (27.6 months; $90 \%$ CI: 25.9-31.3 months; Figure 1). The median time on afatinib treatment was 11.9 months (90\% CI: 10.9-12.2 months) and the median time on osimertinib treatment was 14.3 months (90\% CI: 12.8-15.9 months). The duration of treatment was largely consistent across patient subgroups and was particularly striking in Asian patients ( 46.7 months) and patients with a Del19-activating mutation (30.3 months; Figure 1). The latter observation was of particular interest because the emergence of T790M in Del19-positive tumors is more common (up to 75\% of cases) than in L858R-positive tumors [55]. Therefore, there is a good chance that Del19-positive patients could be eligible for osimertinib after failure of first-line afatinib. Recently, an interim analysis of GioTag OS data was undertaken. After a median follow-up of 30.3 months, median OS was nearly 3.5 years (41.3 months; 90\% CI: 36.8-46.3; Figure 1). The 2 -year OS rate was $80 \%$. In patients with Del19-positive tumors, median OS was even higher (45.7 months; $90 \%$ CI: 45.3-51.5) and the 2-year OS rate was $82 \%$. A final analysis of OS is due in 2020 [56]. These findings suggest 


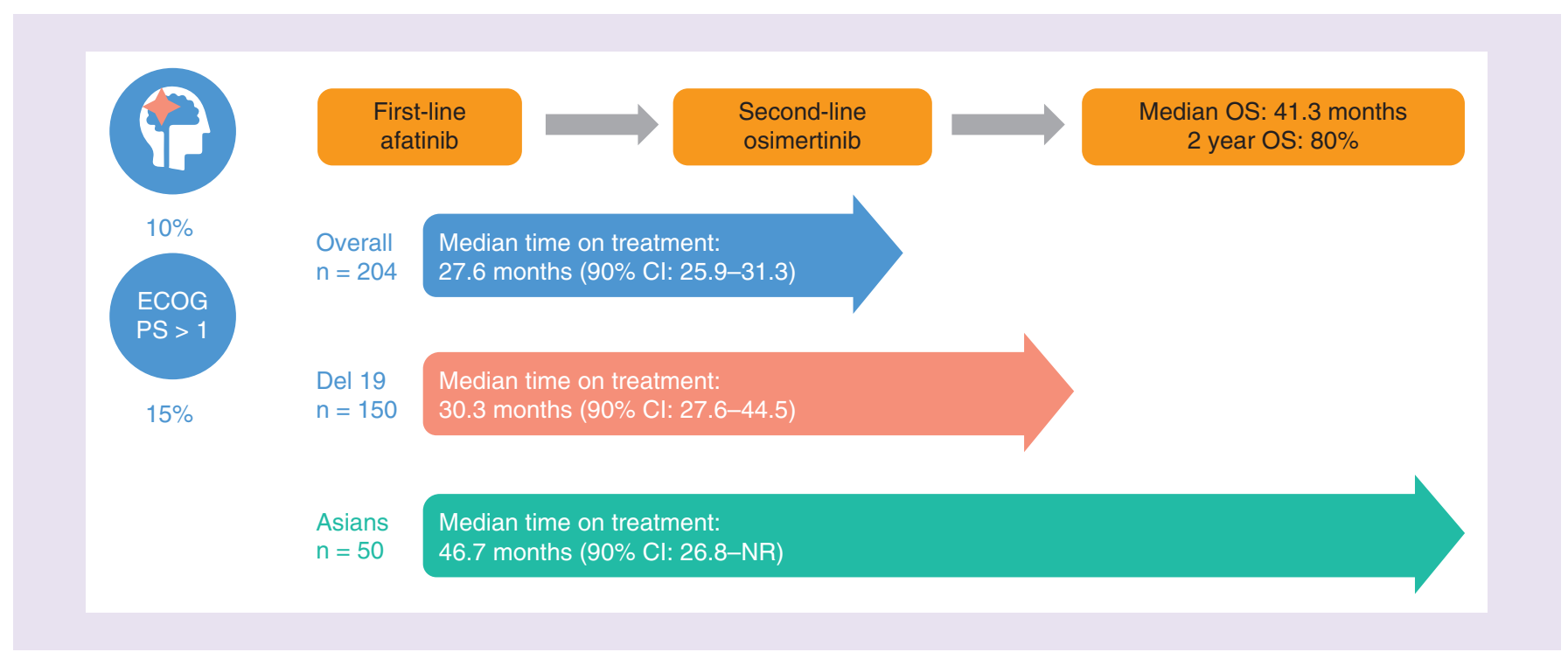

Figure 1. Outcomes in patients treated with sequential afatinib and osimertinib in the observational GioTag study. NR: Not reached.

Park K, Lim DW-T, Okamoto I et al., Therapeutic Advances in Medical Oncology (11) pp. 1-17, copyright (c) 2019 by The Author(s). Reprinted by Permission of SAGE Publications, Ltd.

that sequential use of afatinib and osimertinib could be an attractive option in patients with Del19-positive tumors. Indeed, previous studies have consistently demonstrated that Del19 is a predictive biomarker in patients with EGFR mutation-positive NSCLC treated with EGFR TKIs [57,58]. For example, it was notable that OS benefit with afatinib versus chemotherapy in the LUX-Lung 3 and 6 trials was observed in patients with Del19-positive tumors, but not L858R-positive tumors [32]. Based on such findings, it has been suggested that Del19- and L858R-positive tumors should be considered as different biological disease entities, for which different therapeutic strategies could be considered [58].

In GioTag, median time on treatment was not adversely affected by the age of patients or the presence of baseline brain metastases. Indeed, although brain imaging was not mandatory, only $6.6 \%$ of patients treated with afatinib were reported to have developed brain metastases. Other studies have indicated that both afatinib and osimertinib are active in the central nervous system (CNS) and can cross the blood-brain barrier [18,19,59,60]. Consequently, sequential treatment with these agents could potentially protect against CNS progression, which is important because CNS involvement is a common feature of EGFR mutation-positive NSCLC [61]. Other patient subgroups that have been poorly represented in clinical trials, such as those with ECOG PS of $>2$ (median of 22.2 months) and African-Americans (median of 27.6 months) also remained on treatment for a prolonged period of time.

Other 'real-world' studies have assessed outcomes in patients with EGFR mutation-positive NSCLC who received sequential EGFR TKIs. In a cohort study undertaken in Austria, 49 patients were identified who received sequential afatinib and osimertinib [29]. Thirty six of these patients received afatinib in a first-line setting. The duration of treatment analysis in this study was immature at the time of writing as 24 patients were still receiving osimertinib. Nevertheless, median duration of osimertinib treatment was 14.0 months overall and was not reached in patients who received first-line afatinib (Figure 2). The response rate to osimertinib was high; $75.5 \%$ overall and $77.8 \%$ in patients who received first-line afatinib. It was noteworthy that in this study, which utilized a sensitive droplet-digital PCR technique to detect T790M, response rate was independent of T790M copy number. This indicates that heterogeneous tumors, with low numbers of T790M-positive cells at the point of acquired resistance to afatinib, could still benefit from subsequent osimertinib, thus underpinning the importance of further development of sensitive blood-based mutation detection assays that can be routinely implemented in the clinic. Another retrospective analysis, undertaken in Germany, identified 141 patients with EGFR mutation-positive NSCLC across three centers who received first- or second-generation EGFR TKIs [62]. Only 19 patients went on to receive osimertinib, possibly reflecting its limited availability at the time. Nevertheless, OS was significantly longer in those who received osimertinib (median 67 months) than those who did not (median 23 months; $\mathrm{p}<0.001$ ). 
(A)

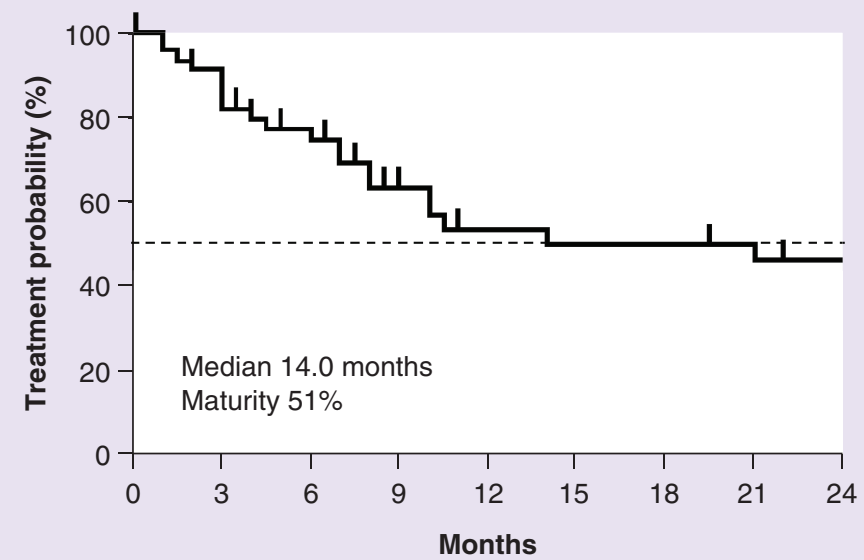

No. at risk:

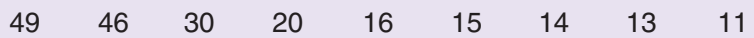

(B)

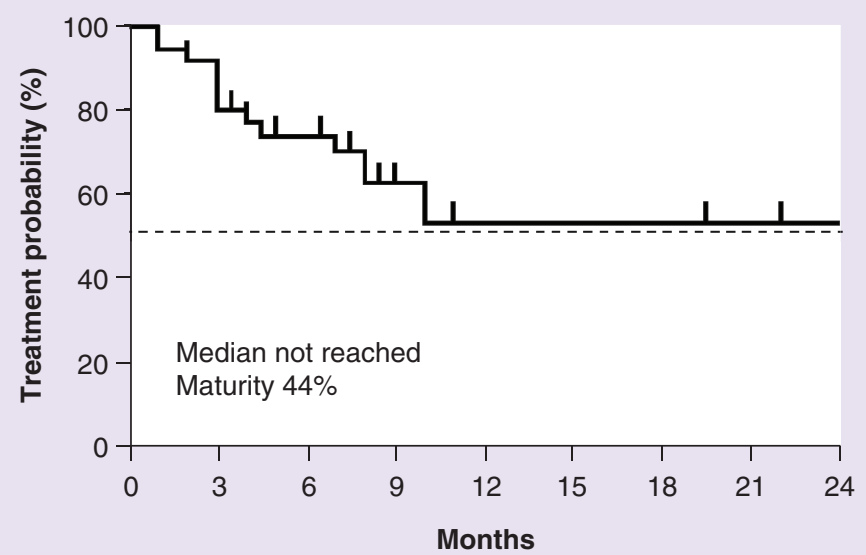

No. at risk:

$\begin{array}{lllllllll}36 & 31 & 22 & 14 & 11 & 11 & 11 & 10 & 9\end{array}$

Figure 2. Duration of osimertinib treatment in a cohort of 49 patients who received sequential afatinib and osimertinib in Austria. (A) All patients; (B) patients who received first-line afatinib.

Reprinted by permission from Springer Nature: Targeted Oncology. Liquid-Biopsy-Based Identification of EGFR T790M Mutation-Mediated Resistance to Afatinib Treatment in Patients with Advanced EGFR Mutation-Positive NSCLC, and Subsequent Response to Osimertinib. Maximilian J. Hochmair, Anna Buder, Sophia Schwab et al., copyright (c) 2018.

Although these studies are retrospective, and do not substitute for prospective trials, they illustrate the potential benefits of sequential EGFR TKIs in patients who develop T790M-positive disease. However, few studies have compared specific sequencing regimens. As described above, the majority of 'real-world' data have assessed sequential afatinib and osimertinib. To the best of current knowledge, no 'real-world' studies have assessed sequential dacomitinib and osimertinib, probably reflecting the fact that afatinib has been available for longer than dacomitinib. In addition, few studies have assessed outcomes with sequential first- and third-generation TKIs compared with second- and third-generation TKIs. Nevertheless, one recent study indicated that the latter sequence might be preferential to the former. In a retrospective study of 111 patients with T790M-positive NSCLC, undertaken in Japan, the objective response rate (ORR) was significantly higher in patients who received afatinib-osimertinib $(82.9 \%)$ than gefitinib/erlotinib-osimertinib $(53.9 \% ; \mathrm{p}=0.0065)$ [63]. PFS was also longer in the former group (median 15.7 vs 8.9 months; $\mathrm{p}=0.195$ ). This observation could reflect the differences in mechanism of action 
of first- and second-generation TKIs, leading to differences in the clonal evolution of tumors. Indeed, recent preclinical data indicate that the copy number of T790M is higher in afatinib-resistant NSCLC tumor cells than erlotinib-resistant tumor cells [64]. This implies that afatinib-treated tumors could be more homogenous than erlotinib-treated tumors (i.e., a greater number of cells are T790M positive) at the point of acquired resistance and are, therefore, more sensitive to subsequent osimertinib. This possibility requires further research.

\section{Sequential regimens \& tolerability considerations}

A key issue when considering optimal treatment with EGFR TKIs is the tolerability profile of different agents, and their suitability in individual patients depending on their general fitness and comorbidity status. While all EGFR TKIs are associated with class-related adverse events (AEs), predominantly gastrointestinal and cutaneous effects, the overall AE profile varies with different generations of TKIs. Owing to the fact that it is wild-type sparing, osimertinib appears to have a better tolerability profile than other EGFR TKIs (though no head-to-head comparisons of tolerability and impact on quality of life exist between osimertinib and second-generation EGFR TKIs). In FLAURA, osimertinib was associated with lower rates of grade $\geq 3 \mathrm{AEs}$ than erlotinib or gefitinib (34 vs $45 \%$ ), with very low rates of treatment-related grade $\geq 3$ diarrhea ( 2 vs $2 \%$ ), rash/acne ( 1 vs $7 \%$ ) and elevated alanine aminotransferase (ALT) $(<1$ vs $8 \%$ ), though certain AEs were higher with osimertinib (e.g., QT elongation: 10 vs 4\%) [11]. Second-generation TKIs, on the other hand, have been associated with higher rates of certain treatmentrelated grade $\geq 3 \mathrm{AEs}$ than first-generation TKIs. In LUX Lung 7, the most common treatment-related grade $\geq 3$ AEs with afatinib versus gefitinib were diarrhea (13 vs $1 \%$ ), rash/acne ( 9 vs $3 \%$ ) and stomatitis ( 4 vs $0 \%$ ) [9]. In ARCHER 1050, the most common grade $\geq 3 \mathrm{AEs}$ with dacomitinib versus gefitinib were dermatitis acneiform (14 vs $0 \%$ ), diarrhea ( 8 vs $1 \%$ ) and paronychia (7 vs 1\%) [10]. These side effects are predictable, and symptoms may be prevented prophylactically.

The lower rates of grade $\geq 3 \mathrm{AEs}$ with osimertinib versus other TKIs, along with the promising activity observed in FLAURA, provide further rationale for utilizing osimertinib as a first-line treatment option. However, it should be noted that AEs with second-generation TKIs are generally manageable and rarely lead to permanent drug discontinuation. Indeed, discontinuation rates due to AEs with osimertinib (13\%), afatinib (11\%) and dacomitinib (10\%) in FLAURA, LUX-Lung 7 and ARCHER 1050 were similar [9-11]. Therefore, there is also a case for initiating treatment with second-generation TKIs, followed by osimertinib in terms of safety, as this could be the best approach for delaying the use of chemotherapy and its associated tolerability/quality-of-life issues for as long as possible. Indeed, in patients with terminal NSCLC, a key consideration is the likely impact of treatment on a patient's health-related quality of life, which is strongly influenced by treatment-related AEs and disease-related symptoms [65]. Over $90 \%$ of patients with advanced NSCLC have at least two disease-related symptoms, such as dyspnea, coughing and pain. These symptoms have such a negative impact on patients' well being that, according to one survey, over two-thirds of patients would prefer a treatment that would improve disease-related symptoms without prolonging their life, than vice versa [65]. As afatinib and osimertinib have both been shown to confer a better tolerability profile, improve symptom control and improve health-related quality of life compared with chemotherapy [66-68], it is reasonable to infer that sequential EGFR TKI therapy could have a net positive impact on patients' overall condition, over a longer time frame, than first-line osimertinib.

Previously reported clinical trial data have indicated that AEs with afatinib can be effectively managed with tolerability-guided dose adjustment and/or supportive care measures, without compromising clinical activity [16,17]. These observations have recently been corroborated by 'real-world' analysis. In the noninterventional RealGiDo study, the medical records of 228 patients with EGFR mutation-positive NSCLC treated with first-line afatinib were reviewed [69]. This cohort was more representative of 'real-world' clinical practice than randomized trials and included patients with poor prognostic features (e.g., 12\% of patients had ECOG PS of $\geq 2$ ). In RealGiDo, 31\% of patients initiated treatment on a reduced dose of $30 \mathrm{mg}$ afatinib. Of the patients who started on the approved dose of $40 \mathrm{mg}$, dose reductions were common (67\%) and predominantly occurred within the first 6 months of treatment (87\%). Dose adjustment effectively reduced the frequency and intensity of AEs, and allowed the majority of patients to remain on treatment for as long as they received clinical benefit. The discontinuation rate due to treatment-related AEs was low (7\%). Dose reduction did not appear to compromise clinical activity. Median time to treatment failure, an end point that assesses both efficacy and tolerability in 'real-world' practice, was similar in patients who remained on $40 \mathrm{mg}$ for the first 6 months, those who had a dose reduction and those who received a starting dose of $30 \mathrm{mg}(19.5,17.7$ and 19.4 months, respectively; $\mathrm{p}=0.543)$. Likewise, time to progression (TTP) was not significantly different between the three groups (29.0, 20.0 and 25.9 months, respectively; $\mathrm{p}=0.392$ ). 
TTP was also consistent across most patient subgroups and was not affected by age or EGFR mutation type. Unsurprisingly, TTP was lower in patients with ECOG PS $\geq 2$ versus those with PS $\leq 1$. To date, no data have been published that have assessed the tolerability of dacomitinib in 'real-world' clinical practice. However, post-hoc analysis of clinical trial data suggested similar findings as for afatinib; dose reduction did not appear to compromise antitumor efficacy but effectively reduced the frequency and severity of AEs [70,71].

Overall, therefore, despite having poorer tolerability profiles in clinical trials, second-generation EGFR TKIs appear to be a viable first-line treatment choice in everyday clinical practice. By monitoring for AEs, which are largely predictable, and employing dose-adjustment protocols, most patients can remain on treatment for as long as they achieve clinical benefit.

\section{Treatment options for patients with T790M-negative NSCLC}

Although there is accumulating evidence to suggest that reserving osimertinib for second-line use is a viable option, one key potential drawback of this approach is that although T790M is the predominant mechanism of acquired resistance to first- and second-generation EGFR TKIs, a proportion of patients will inevitably progress due to T790M-independent mechanisms. These patients will not be eligible for osimertinib. Targeted treatment options for T790M-negative tumors remain an area of unmet need, and most patients who go on to receive second-line treatment will receive chemotherapy [72]. Nevertheless, several potential treatment modalities in this setting are beginning to emerge.

The recent Phase III IMpower 150 trial assessed the efficacy and safety of the anti-PD-L1 antibody, atezolizumab, combined with platinum-doublet chemotherapy and bevacizumab in patients with chemotherapy-naive NSCLC [73]. Although previous immunotherapy drug trials had yielded disappointing results in patients with EGFR mutation-positive tumors, IMpower 150 permitted the enrollment of such patients, most of whom had received an EGFR TKI. Of interest, the atezolizumab-containing arm conferred significantly higher PFS and OS than the control arm (chemotherapy plus bevacizumab) in patients with sensitive EGFR mutations (median PFS: 10.3 vs 6.1 months; HR: 0.41 ; 95\% CI: 0.23-0.75; median OS: not reached vs 17.5 months; HR: 0.31 ; $95 \%$ CI: $0.11-0.83)$ [74]. These exciting findings suggest that immunotherapy combinations could have a role in patients EGFR mutation-positive tumors following failure of first-line TKIs. An ongoing Phase III trial is assessing the combination of pembrolizumab with chemotherapy in patients with EGFR mutation-positive tumors resistant or refractory to EGFR TKIs (NCT03515837). Another trial is assessing the combinations of nivolumab and ipilimumab or chemotherapy plus nivolumab in the same setting and after the failure of osimertinib first or second line (NCT02864251).

Another potential option in patients with T790M-negative tumors could be EGFR TKI-based combination regimens. The Phase III IMPRESS trial assessed gefitinib plus chemotherapy in patients with EGFR mutationpositive NSCLC who had progressed on gefitinib monotherapy. In patients with T790M-negative tumors, there was a trend toward improved PFS versus chemotherapy alone (6.7 vs 5.4 months; HR: 0.67; 95\% CI: 0.43-1.03; $\mathrm{p}=0.0745)$ [75]. Furthermore, in a Phase II trial, the combination of erlotinib and bevacizumab conferred median PFS of 10.5 months and ORR of $79 \%$ in 72 patients with T790M-negative tumors [76]. In the recent ABC Phase II study, the combination of afatinib and bevacizumab demonstrated promising activity with median PFS of 7.1 months and ORR of $22 \%$ in 18 patients with T790M-negative tumors [77].

Emerging data suggest that rechallenge with EGFR TKIs could have a role in patients with T790M-negative tumors. In a recent Phase II trial, 12 patients with acquired resistance to first- or second-generation EGFR TKIs were treated with cytotoxic agents followed by afatinib [78]. Of note, afatinib demonstrated modest clinical activity in this setting, with an ORR of $17 \%$ and median PFS of 4.2 months. Further studies are, therefore, warranted to further assess the concept of EGFR TKI rechallenge.

\section{Conclusion}

Recent head-to-head trials have demonstrated that second- and third-generation EGFR TKIs are preferable to first-generation EGFR TKIs as first-line treatment for patients with EGFR mutation-positive NSCLC. However, given the absence of prospective data, it is not clear whether it is preferable to initiate treatment with a second- or third-generation TKI. There are arguments for both approaches. FLAURA demonstrated that first-line osimertinib is very active in a first-line setting, with a favorable tolerability profile [11]. Furthermore, analysis of those patients who received a subsequent EGFR TKI indicated that PFS-2 was superior in the osimertinib arm compared with the erlotinib/gefitinib arm [43]. On the other hand, treatment options following osimertinib are not clear and most 
patients who receive subsequent therapy receive chemotherapy. Therefore, it may be preferable to reserve osimertinib for second-line use especially as T790M appears to be the predominant mechanism of resistance to afatinib (few data are available regarding resistance mechanisms to dacomitinib). Recent 'real-world' data, notably the observational GioTag study, and subanalysis of the LUX-Lung and ARCHER 1050 trials demonstrate that sequential secondgeneration EGFR TKIs and osimertinib can effectively provide prolonged periods of chemotherapy-free treatment in patients with T790M-mediated resistance. Ultimately, prospective trials are required to compare different sequential regimens with $O S$ as a primary end point.

\section{Future perspective}

Since a previous review [18], several developments have provided supportive evidence for the application of sequential second- and third-line EGFR TKI therapy. Such an approach could help to maximize OS in patients with EGFR mutation-positive NSCLC who acquire T790M. In the future, optimization of therapy will require further improvements in the monitoring of the molecular evolution of tumors, and mechanisms of resistance, over the course of several lines of therapy. This could facilitate a 'precision medicine' approach where treatment is tailored according to the molecular evolution of the tumor. Another area of progress appears to be the optimization of first-line EGFR TKIs by combining with antiangiogenic agents, including bevacizumab $[12,13]$ and, more recently, ramucirumab [14]. Importantly, combination of EGFR TKIs with other agents does not appear to preclude access to subsequent osimertinib. For instance, T790M-positive resistance was observed in nearly half the patients treated with erlotinib plus ramucirumab.

At the moment, there are no prospective data that have compared OS between different sequential EGFR TKI regimens. Final OS data from FLAURA and AURA3 will be informative, as will other ongoing trials (e.g., APPLE). However, only AURA3 included patients who received first-line afatinib. Therefore, more trials need to be designed and undertaken. With a personalized medicine approach, it is likely that different sequential approaches will be required according to individual patient and disease characteristics.

Regarding the treatment of EGFR mutation-positive NSCLC, a number of areas of unmet medical need are still to be addressed. As discussed, a key need is effective targeted treatment options following the failure of osimertinib. A number of possibilities are currently being investigated. Clearly, further information is required regarding the molecular mechanisms of resistance to osimertinib, as well as standardized assays that can be applied to identify these mechanisms in everyday clinical practice. Finally, targeted treatment options for patients with T790M-independent mechanisms of resistance need to be identified. With further progress in these areas, it is anticipated that in the future EGFR mutation-positive NSCLC could be regarded as a chronic disease in many patients.

\section{Executive summary}

Mechanisms of resistance to EGFR tyrosine kinase inhibitors

- The emergence of T790M is the predominant mechanism of resistance to first-generation EGFR tyrosine kinase inhibitors (TKIs) in patients with EGFR mutation-positive non-small-cell lung cancer (NSCLC).

- Recent clinical trial and 'real-world' data demonstrate that T790M is also the most common resistance mechanism to afatinib.

- Resistance mechanisms to osimertinib appear to be more heterogeneous than those to first- and second-generation EGFR TKIs. In the majority of patients, putative mechanisms of resistance have not been identified.

- Currently no standard targeted therapy options exist following failure of osimertinib and most patients receive chemotherapy. However, several targeted agents have recently shown promising efficacy following osimertinib in early phase clinical trials.

Subsequent therapy after first-line TKIs: lessons from clinical trials

- Post-hoc analysis of clinical trials indicate that most patients treated with second-generation EGFR TKIs are fit enough to receive subsequent therapy.

- Few patients treated with afatinib or dacomitinib in clinical trials received subsequent osimertinib, reflecting its lack of availability at the time. Nevertheless, overall survival (OS) in these patients was highly encouraging.

- In FLAURA, 59\% of patients received subsequent therapy following failure of osimertinib. Analysis of PFS-2 and time to discontinuation or death suggest that osimertinib preserves clinical benefit beyond first progression versus first-generation EGFR TKIs, but OS data are immature.

- Prospective data will be required to determine the optimal sequence of EGFR TKIs in patients with EGFR mutation-positive NSCLC. 
Subsequent therapy after first-line TKIs: lessons from 'real-world' data

- Several 'real-world' studies have demonstrated encouraging clinical outcomes in patients receiving sequential EGFR TKIs.

- In the observational GioTag study, a broad cohort of 204 patients with treatment-naive EGFR mutation-positive NSCLC received sequential afatinib and osimertinib. Median time on treatment was 27.6 months overall, 30.3 months in patients with Del19 mutation-positive tumors and 46.7 months in Asian patients. Median OS was 41.3 months and the 2 -year OS rate was $80 \%$.

- Other 'real-world' studies, including cohort studies undertaken in Austria and Germany, illustrate the potential benefits of sequential afatinib and osimertinib in patients who develop T790M-positive disease.

- Such an approach will require rebiopsy of tumor samples at the time of acquired resistance. Development of sensitive blood-based assays may improve accessibility to sequential EGFR TKI regimens in everyday clinical practice.

Sequential regimens \& tolerability considerations

- Owing to the fact that it is wild-type sparing, osimertinib appears to have a better tolerability profile than other EGFR TKIs.

- Second-generation TKIs have been associated with higher rates of certain treatment-related grade $\geq 3$ adverse events, such as diarrhea and rash/acne, compared with first-generation TKIs.

- These side effects are predictable, may be prevented prophylactically and can be managed with dose adjustments without affecting treatment efficacy; hence, dose discontinuations due to treatment-related AEs are low.

- Recent 'real-world' observations, including the noninterventional RealGiDo study, indicate that afatinib is tolerable in patients treated in a standard clinical practice setting.

Treatment options for patients with T790M-negative NSCLC

- One key drawback of reserving osimertinib for second-line use is that a proportion of patients, that is, those with T790M-negative acquired resistance, may never benefit from its efficacy and safety benefits.

- While certain treatment modalities, for example, immunotherapy combinations, have shown promise in this setting, targeted treatment options for patients with T790M-negative disease remain an unmet clinical need.

Conclusion

- Based on the FLAURA trial, there is a strong rationale for considering first-line osimertinib in patients with EGFR mutation-positive NSCLC, given its favorable efficacy and safety.

- However, given the current paucity of targeted treatment options following its failure, it may be preferable to reserve osimertinib for second-line use, where it has also shown strong activity in patients with T790M-positive disease.

- The majority of tumors treated with second-generation TKIs could potentially receive second-line osimertinib, subject to a positive T790M test.

- Recent 'real-world' data, notably the observational GioTag study, and subanalysis of the LUX-Lung and ARCHER 1050 trials, demonstrate that sequential second-generation EGFR TKIs and osimertinib can effectively provide prolonged periods of chemotherapy-free treatment in patients with T790M-mediated resistance.

- Ultimately, prospective trials are required to compare different sequential regimens with OS as a primary end point.

Financial \& competing interests disclosure

N Girard has acted as a consultant and received financial assistance for travel from AstraZeneca, Hoffman LaRoche, Boehringer Ingelheim and Pfizer. He has also received academic grants from AstraZeneca, Hoffman LaRoche, Boehringer Ingelheim and Pfizer. The author has no other relevant affiliations or financial involvement with any organization or entity with a financial interest in or financial conflict with the subject matter or materials discussed in the manuscript apart from those disclosed.

Medical writing assistance, financially supported by Boehringer Ingelheim, was provided by L Pritchard of GeoMed, an Ashfield company, part of UDG Healthcare plc, during the preparation of this article.

\section{Open access}

This work is licensed under the Attribution-NonCommercial-NoDerivatives 4.0 Unported License. To view a copy of this license, visit http://creativecommons.org/licenses/by-nc-nd/4.0/

Supplementary data

An infographic accompanies this paper at the end of the references section. To download the infographic that accompanies this paper, please visit the journal website at: https://www.futuremedicine.com/pb-assets/Infographics/1128935_Girard-sequencingreview-infographic_v1f-1568909283830.pdf 


\section{References}

Papers of special note have been highlighted as: $\bullet$ of interest; $\bullet \bullet$ of considerable interest

1. Mok TS, Wu YL, Thongprasert S et al. Gefitinib or carboplatin-paclitaxel in pulmonary adenocarcinoma. N. Engl. J. Med. 361 947-957(2009).

2. Maemondo M, Inoue A, Kobayashi $\mathrm{K}$ et al. Gefitinib or chemotherapy for non-small-cell lung cancer with mutated EGFR. N. Engl. J. Med. 362, 2380-2388 (2010).

3. Mitsudomi T, Morita S, Yatabe Y et al. Gefitinib versus cisplatin plus docetaxel in patients with non-small-cell lung cancer harbouring mutations of the epidermal growth factor receptor (WJTOG3405): an open label, randomised Phase III trial. Lancet Oncol. 11, 121-128 (2010).

4. Zhou C, Wu YL, Chen G et al. Erlotinib versus chemotherapy as first-line treatment for patients with advanced EGFR mutation-positive non-small-cell lung cancer (OPTIMAL, CTONG-0802): a multicentre, open-label, randomised, Phase III study. Lancet Oncol. 12, 735-742(2011).

5. Rosell R, Carcereny E, Gervais R et al. Erlotinib versus standard chemotherapy as first-line treatment for European patients with advanced EGFR mutation-positive non-small-cell lung cancer (EURTAC): a multicentre, open-label, randomised Phase III trial. Lancet Oncol. 13, 239-246 (2012).

6. Sequist LV, Yang JC, Yamamoto N et al. Phase III study of afatinib or cisplatin plus pemetrexed in patients with metastatic lung adenocarcinoma with EGFR mutations. J. Clin. Oncol. 31, 3327-3334 (2013).

7. Wu YL, Zhou C, Hu CP et al. Afatinib versus cisplatin plus gemcitabine for first-line treatment of Asian patients with advanced non-small-cell lung cancer harbouring EGFR mutations (LUX-Lung 6): an open-label, randomised Phase III trial. Lancet Oncol. 15, 213-222 (2014).

8. Wu YL, Zhou C, Liam CK et al. First-line erlotinib versus gemcitabine/cisplatin in patients with advanced EGFR mutation-positive non-small-cell lung cancer: analyses from the Phase III, randomized, open-label, ENSURE study. Ann. Oncol. 26, 1883-1889 (2015).

9. Park K, Tan EH, O’Byrne K et al. Afatinib versus gefitinib as first-line treatment of patients with EGFR mutation-positive non-small-cell lung cancer (LUX-Lung 7): a Phase IIB, open-label, randomised controlled trial. Lancet Oncol. 17, 577-589 (2016).

10. Wu YL, Cheng Y, Zhou X et al. Dacomitinib versus gefitinib as first-line treatment for patients with EGFR-mutation-positive non-small-cell lung cancer (ARCHER 1050): a randomised, open-label, Phase III trial. Lancet Oncol. 18, 1454-1466 (2017).

11. Soria JC, Ohe Y, Vansteenkiste J et al. Osimertinib in untreated EGFR-mutated advanced non-small-cell lung cancer. N. Engl. J Med. 378, 113-125 (2018).

12. Seto T, Kato T, Nishio $\mathrm{M}$ et al. Erlotinib alone or with bevacizumab as first-line therapy in patients with advanced non-squamous non-small-cell lung cancer harbouring EGFR mutations (JO25567): an open-label, randomised, multicentre, Phase II study. Lancet Oncol. 15, 1236-1244 (2014).

13. Saito H, Fukuhara T, Furuya $\mathrm{N}$ et al. Erlotinib plus bevacizumab versus erlotinib alone in patients with EGFR-positive advanced non-squamous non-small-cell lung cancer (NEJ026): interim analysis of an open-label, randomised, multicentre, Phase III trial. Lancet Oncol. 20, 625-635 (2019).

14. Nakagawa K, Garon EB, Seto T et al. RELAY: A multinational, double-blind, randomized Phase III study of erlotinib (ERL) in combination with ramucirumab (RAM) or placebo (PL) in previously untreated patients with epidermal growth factor receptor mutation-positive (EGFRm) metastatic non-small cell lung cancer (NSCLC). J. Clin. Oncol. 37, 9000-9000 (2019).

15. Nakamura A, Inoue A, Morita $S$ et al. Phase III study comparing gefitinib monotherapy $(G)$ to combination therapy with gefitinib, carboplatin, and pemetrexed (GCP) for untreated patients (pts) with advanced non-small cell lung cancer (NSCLC) with EGFR mutations (NEJ009). J. Clin. Oncol. 36, 9005-9005 (2018).

16. Yang JC, Sequist LV, Zhou C et al. Effect of dose adjustment on the safety and efficacy of afatinib for EGFR mutation-positive lung adenocarcinoma: post hoc analyses of the randomized LUX-Lung 3 and 6 trials. Ann. Oncol. 27, 2103-2110 (2016).

17. Schuler M, Tan EH, O'Byrne K et al. First-line afatinib vs gefitinib for patients with EGFR mutation-positive NSCLC (LUX-Lung 7): impact of afatinib dose adjustment and analysis of mode of initial progression for patients who continued treatment beyond progression. J. Cancer Res. Clin. Oncol. 145, 1569-1579 (2019).

18. Girard N. Optimizing outcomes in EGFR mutation-positive NSCLC: which tyrosine kinase inhibitor and when? Future Oncol. 14 , $1117-1132(2018)$.

19. Reungwetwattana $\mathrm{T}$, Nakagawa $\mathrm{K}$, Cho BC et al. CNS response to osimertinib versus standard epidermal growth factor receptor tyrosine kinase inhibitors in patients with untreated EGFR-mutated advanced non-small-cell lung cancer. J. Clin. Oncol. 36, 3290-3297 (2018).

20. Schuler M, Wu YL, Hirsh V et al. First-line afatinib versus chemotherapy in patients with non-small cell lung cancer and common epidermal growth factor receptor gene mutations and brain metastases. J. Thorac. Oncol. 11, 380-390 (2016).

21. Hirsh V. Turning EGFR mutation-positive non-small-cell lung cancer into a chronic disease: optimal sequential therapy with EGFR tyrosine kinase inhibitors. Ther. Adv. Med. Oncol. 10, 1-12 (2018)

22. Sun JM, Park K. Can we define the optimal sequence of epidermal growth factor receptor tyrosine kinase inhibitors for the treatment of epidermal growth factor receptor-mutant nonsmall cell lung cancer? Curr. Opin. Oncol. 29, 89-96 (2017). 
23. Oxnard GR, Hu Y, Mileham KF et al. Assessment of resistance mechanisms and clinical implications in patients with EGFR T790M-positive lung cancer and acquired resistance to osimertinib. JAMA Oncol. 4, 1527-1534 (2018).

24. Ramalingam SS, Cheng Y, Zhou C et al. Mechanisms of acquired resistance to first-line osimertinib: preliminary data from the Phase III FLAURA study. Ann. Oncol. 29, viii740 (2018).

25. Papadimitrakopoulou VA, Wu Y-L, Han J-Y et al. Analysis of resistance mechanisms to osimertinib in patients with EGFR T790M advanced NSCLC from the AURA3 study. Ann. Oncol. 29, viii741 (2018).

26. Janne PA, Yang JC, Kim DW et al. AZD9291 in EGFR inhibitor-resistant non-small-cell lung cancer. N. Engl. J. Med. 372, 1689-1699 (2015).

27. Mok TS, Wu YL, Ahn MJ et al. Osimertinib or platinum-pemetrexed in EGFR T790M-positive lung cancer. N. Engl. J. Med. 376, 629-640 (2017).

28. Yang JC, Ahn MJ, Kim DW et al. Osimertinib in pretreated T790M-positive advanced non-small-cell lung cancer: AURA study Phase II extension component. J. Clin. Oncol. 35, 1288-1296 (2017).

29. Hochmair MJ, Buder A, Schwab S et al. Liquid-biopsy-based identification of EGFR T790M mutation-mediated resistance to afatinib treatment in patients with advanced EGFR mutation-positive NSCLC, and subsequent response to osimertinib. Target. Oncol. 14, 75-83 (2019).

- A retrospective analysis shows a T790M emergence rate of $73 \%$ in 67 patients treated with afatinib, based on a highly sensitive blood biopsy assay.

30. Mok TS, Cheng Y, Zhou X et al. Improvement in overall survival in a randomized study that compared dacomitinib with gefitinib in patients with advanced non-small-cell lung cancer and EGFR-activating mutations. J. Clin. Oncol. 36, 2244-2250 (2018).

- Exploratory analysis of the Phase III ARCHER 1050 trial demonstrates that the ErbB family blocker, dacomitinib, improved overall survival versus gefitinib in patients with EGFR mutation-positive non-small-cell lung cancer.

31. Paz-Ares L, Tan EH, O’Byrne $\mathrm{K}$ et al. Afatinib versus gefitinib in patients with EGFR mutation-positive advanced non-small-cell lung cancer: overall survival data from the Phase IIb LUX-Lung 7 trial. Ann. Oncol. 28, 270-277 (2017).

32. Yang JC, Wu YL, Schuler M et al. Afatinib versus cisplatin-based chemotherapy for EGFR mutation-positive lung adenocarcinoma (LUX-Lung 3 and LUX-Lung 6): analysis of overall survival data from two randomised, Phase III trials. Lancet Oncol. 16, 141-151 (2015).

33. Park K, Bennouna J, Boyer M et al. Sequencing of therapy following first-line afatinib in patients with EGFR mutation-positive non-small cell lung cancer. Lung Cancer 132, 126-131 (2019).

- Retrospective analysis of patients treated with sequential afatinib and osimertinib in the randomized LUX-Lung 3, 6 and 7 trials.

34. Hochmair MJ. How can better identification of T790M help to inform treatment sequencing decisions in EGFR mutation-positive non-small-cell lung cancer? Future Oncol. doi:10.2217/fon-2019-0142 (2019) (Epub ahead of print).

35. Arcila ME, Oxnard GR, Nafa K et al. Rebiopsy of lung cancer patients with acquired resistance to EGFR inhibitors and enhanced detection of the T790M mutation using a locked nucleic acid-based assay. Clin. Cancer Res. 17, 1169-1180 (2011).

36. Sequist LV, Waltman BA, Dias-Santagata D et al. Genotypic and histological evolution of lung cancers acquiring resistance to EGFR inhibitors. Sci. Transl. Med. 3, 75 ra26 (2011).

37. Liang SK, Hsieh MS, Lee MR et al. Real-world experience of afatinib as a first-line therapy for advanced EGFR mutation-positive lung adenocarcinoma. Oncotarget 8, 90430-90443 (2017).

38. Tanaka K, Nosaki K, Otsubo K et al. Acquisition of the T790M resistance mutation during afatinib treatment in EGFR tyrosine kinase inhibitor-naive patients with non-small-cell lung cancer harboring EGFR mutations. Oncotarget 8, 68123-68130 (2017).

39. Wu SG, Liu YN, Tsai MF et al. The mechanism of acquired resistance to irreversible EGFR tyrosine kinase inhibitor-afatinib in lung adenocarcinoma patients. Oncotarget 7, 12404-12413 (2016).

40. Kobayashi Y, Fujino T, Nishino M et al. EGFR T790M and C797S mutations as mechanisms of acquired resistance to dacomitinib. J. Thorac. Oncol. 13, 727-731 (2018).

41. Shepherd FA, Papadimitrakopoulou V, Mok T et al. Early clearance of plasma EGFR mutations as a predictor of response to osimertinib in the AURA3 trial. J. Clin. Oncol. 36, 9027-9027 (2018).

42. Schoenfeld AJ, Chan JM, Rizvi H et al. Tissue-based molecular and histological landscape of acquired resistance to osimertinib given initially or at relapse in patients with EGFR-mutant lung cancers. J. Clin. Oncol. 37, 9028-9028 (2019).

43. Planchard D, Boyer MJ, Lee JS et al. Postprogression outcomes for osimertinib versus standard-of-care EGFR-TKI in patients with previously untreated EGFR-mutated advanced non-small cell lung cancer. Clin. Cancer Res. 25, 2058-2063 (2019).

44. Giroux-Leprieur E, Dumenil C, Chinet T. Combination of crizotinib and osimertinib or erlotinib might overcome MET-mediated resistance to EGFR tyrosine kinase inhibitor in EGFR-mutated adenocarcinoma. J. Thorac. Oncol. 13, e232-e234 (2018).

45. Piotrowska Z, Isozaki H, Lennerz JK et al. Landscape of acquired resistance to osimertinib in EGFR-mutant NSCLC and clinical validation of combined EGFR and RET inhibition with osimertinib and BLU-667 for acquired RET fusion. Cancer Discov. 8, 1529-1539 (2018). 
46. Jia Y, Yun CH, Park E et al. Overcoming EGFR(T790M) and EGFR(C797S) resistance with mutant-selective allosteric inhibitors. Nature 534, 129-132 (2016).

47. Moores SL, Chiu ML, Bushey BS et al. A novel bispecific antibody targeting EGFR and cMet is effective against EGFR inhibitor-resistant lung tumors. Cancer Res. 76, 3942-3953 (2016).

48. Haura EB, Cho BC, Lee JS et al. JNJ-61186372 (JNJ-372), an EGFR-cMet bispecific antibody, in EGFR-driven advanced non-small-cell lung cancer (NSCLC). J. Clin. Oncol. 37, 9009-9009 (2019).

49. Janne PA, Yu HA, Johnson ML et al. Safety and preliminary antitumor activity of U3-1402: a HER3-targeted antibody drug conjugate in EGFR TKI-resistant, EGFRm NSCLC. J. Clin. Oncol. 37, 9010-9010 (2019).

50. Ahn MJ, Tsai CM, Shepherd FA et al. Osimertinib in patients with T790M mutation-positive, advanced non-small cell lung cancer: long-term follow-up from a pooled analysis of 2 Phase II studies. Cancer 125, 892-901 (2019).

51. Remon J, Menis J, Hasan B et al. The APPLE trial: feasibility and activity of AZD9291 (osimertinib) treatment on positive plasma T790M in EGFR-mutant NSCLC patients. EORTC 1613. Clin. Lung Cancer 18, 583-588 (2017).

52. Khozin S, Blumenthal GM, Pazdur R. Real-world data for clinical evidence generation in oncology. J. Natl Cancer Inst. 109, djx187 (2017).

53. Sherman RE, Anderson SA, Dal Pan GJ et al. Real-world evidence - what is it and what can it tell us? N. Engl. J. Med. 375, 2293-2297 (2016).

54. Hochmair MJ, Morabito A, Hao D et al. Sequential treatment with afatinib and osimertinib in patients with EGFR mutation-positive non-small-cell lung cancer: an observational study. Future Oncol. 14, 2861-2874 (2018).

-• Primary analysis of the observational GioTag study demonstrates highly encouraging time-to-treatment failure in 204 patients treated with sequential afatinib and osimertinib, especially in those with Del19-positive tumors or Asian ethnicity.

55. Jenkins S, Chih-Hsin Yang J, Janne PA et al. EGFR mutation analysis for prospective patient selection in two Phase II registration studies of osimertinib. J. Thorac. Oncol. 12, 1247-1256 (2017).

56. Hochmair MJ, Morabito A, Hao D et al. Sequential afatinib and osimertinib in patients with EGFR mutation-positive non-small-cell lung cancer: updated analysis of the observational GioTag study. Future Oncol. doi:10.2217/fon-2019-0346 (2019) (Epub ahead of print).

-. Updated analysis from the observational GioTag study which shows median overall survival of 41.3 months in 203 patients treated with sequential afatinib and osimertinib.

57. Lee CK, Wu YL, Ding PN et al. Impact of specific epidermal growth factor receptor (EGFR) mutations and clinical characteristics on outcomes after treatment with EGFR tyrosine kinase inhibitors versus chemotherapy in EGFR-mutant lung cancer: a meta-analysis. $J$. Clin. Oncol. 33, 1958-1965 (2015).

58. Reguart N, Remon J. Common EGFR-mutated subgroups (Del19/L858R) in advanced non-small-cell lung cancer: chasing better outcomes with tyrosine kinase inhibitors. Future Oncol. 11, 1245-1257 (2015).

59. Hoffknecht $\mathrm{P}$, Tufman A, Wehler T et al. Efficacy of the irreversible ErbB family blocker afatinib in epidermal growth factor receptor (EGFR) tyrosine kinase inhibitor (TKI)-pretreated non-small-cell lung cancer patients with brain metastases or leptomeningeal disease. J. Thorac. Oncol. 10, 156-163 (2015).

60. Tamiya A, Tamiya M, Nishihara T et al. Cerebrospinal fluid penetration rate and efficacy of afatinib in patients with EGFR mutation-positive non-small cell lung cancer with leptomeningeal carcinomatosis: a multicenter prospective study. Anticancer Res. 37, 4177-4182 (2017).

61. Hochmair M. Medical treatment options for patients with epidermal growth factor receptor mutation-positive non-small cell lung cancer suffering from brain metastases and/or leptomeningeal disease. Target. Oncol. 13, 269-285 (2018).

62. Roeper J, Lueers A, Netchaeva M et al. Impact on OS and PFS of 2nd and 3rd generation TKI in EGFR mt+ and ALK+ pts: results of the NOWEL network. Ann. Oncol. 28, 486 (2017).

63. Tamiya M, Tamiya A, Suzuki H et al. Which is better EGFR-TKI followed by osimertinib: afatinib or gefitinib/erlotinib? Anticancer Res. 39, 3923-3929 (2019).

- A retrospective analysis suggests that afatinib followed by osimertinib may provide better outcomes for T790M-positive non-small-cell lung cancer than first-generation EGFR tyrosine kinase inhibitors followed by afatinib.

64. Kohsaka S, Petronczki M, Solca F, Maemondo M. Tumor clonality and resistance mechanisms in EGFR mutation-positive non-small-cell lung cancer: implications for therapeutic sequencing. Future Oncol. 15, 637-652 (2019).

65. Hirsh V. Is the evaluation of quality of life in NSCLC trials important? Are the results to be trusted? Front. Oncol. 4, 173 (2014).

66. Geater SL, Xu CR, Zhou C et al. Symptom and quality of life improvement in LUX-Lung 6: an open-label Phase III study of afatinib versus cisplatin/gemcitabine in Asian patients with EGFR mutation-positive advanced non-small-cell lung cancer. J. Thorac. Oncol. 10, 883-889 (2015).

67. Yang JC, Hirsh V, Schuler M et al. Symptom control and quality of life in LUX-Lung 3: a Phase III study of afatinib or cisplatin/pemetrexed in patients with advanced lung adenocarcinoma with EGFR mutations. J. Clin. Oncol. 31, 3342-3350 (2013). 
68. Lee CK, Novello S, Ryden A et al. Patient-reported symptoms and impact of treatment with osimertinib versus chemotherapy in advanced non-small-cell lung cancer: the AURA3 trial. J. Clin. Oncol. 36, 1853-1860 (2018).

69. Halmos B, Tan EH, Soo RA et al. Impact of afatinib dose modification on safety and effectiveness in patients with EGFR mutation-positive advanced NSCLC: results from a global real-world study (RealGiDo). Lung Cancer 127, 103-111 (2019).

70. Mok T, Nakagawa K, Rosell R et al. MA26.11 effects of dose modifications on the safety and efficacy of dacomitinib for EGFR mutation-positive NSCLC. J. Thorac. Oncol. 13, S454 (2018).

71. Zhou Q, Wu Y-L, Corral J et al. Management of common adverse events related to first-line dacomitinib use in EGFR mutation-positive non-small-cell lung cancer: a pooled safety analysis. Future Oncol. 15, 1481-1491 (2019).

72. Corallo S, D’Argento E, Strippoli A et al. Treatment options for EGFR T790M-negative EGFR tyrosine kinase inhibitor-resistant non-small-cell lung cancer. Target. Oncol. 12, 153-161 (2017).

73. Socinski MA, Jotte RM, Cappuzzo F et al. Atezolizumab for first-line treatment of metastatic nonsquamous NSCLC. N. Engl. J. Med. 378, 2288-2301 (2018).

74. Reck M, Mok TSK, Nishio M et al. Atezolizumab plus bevacizumab and chemotherapy in non-small-cell lung cancer (IMpower150): key subgroup analyses of patients with EGFR mutations or baseline liver metastases in a randomised, open-label Phase III trial. Lancet Respir. Med. 7, 387-401 (2019).

75. Mok TSK, Kim SW, Wu YL et al. Gefitinib plus chemotherapy versus chemotherapy in epidermal growth factor receptor mutation-positive non-small-cell lung cancer resistant to first-line gefitinib (IMPRESS): overall survival and biomarker analyses. J. Clin. Oncol. 35, 4027-4034 (2017).

76. Rosell R, Dafni U, Felip E et al. Erlotinib and bevacizumab in patients with advanced non-small-cell lung cancer and activating EGFR mutations (BELIEF): an international, multicentre, single-arm, Phase II trial. Lancet Respir. Med. 5, 435-444 (2017).

77. Hata A, Katakami N, Kaji R et al. Afatinib plus bevacizumab combination after acquired resistance to EGFR tyrosine kinase inhibitors in EGFR-mutant non-small-cell lung cancer: multicenter, single-arm, Phase II trial (ABC Study). Cancer 124, 3830-3838 (2018).

78. Oda N, Hotta K, Ninomiya K et al. A Phase II trial of EGFR-TKI readministration with afatinib in advanced non-small-cell lung cancer harboring a sensitive non-T790M EGFR mutation: Okayama Lung Cancer Study Group trial 1403. Cancer Chemother. Pharmacol. 82, 1031-1038 (2018). 

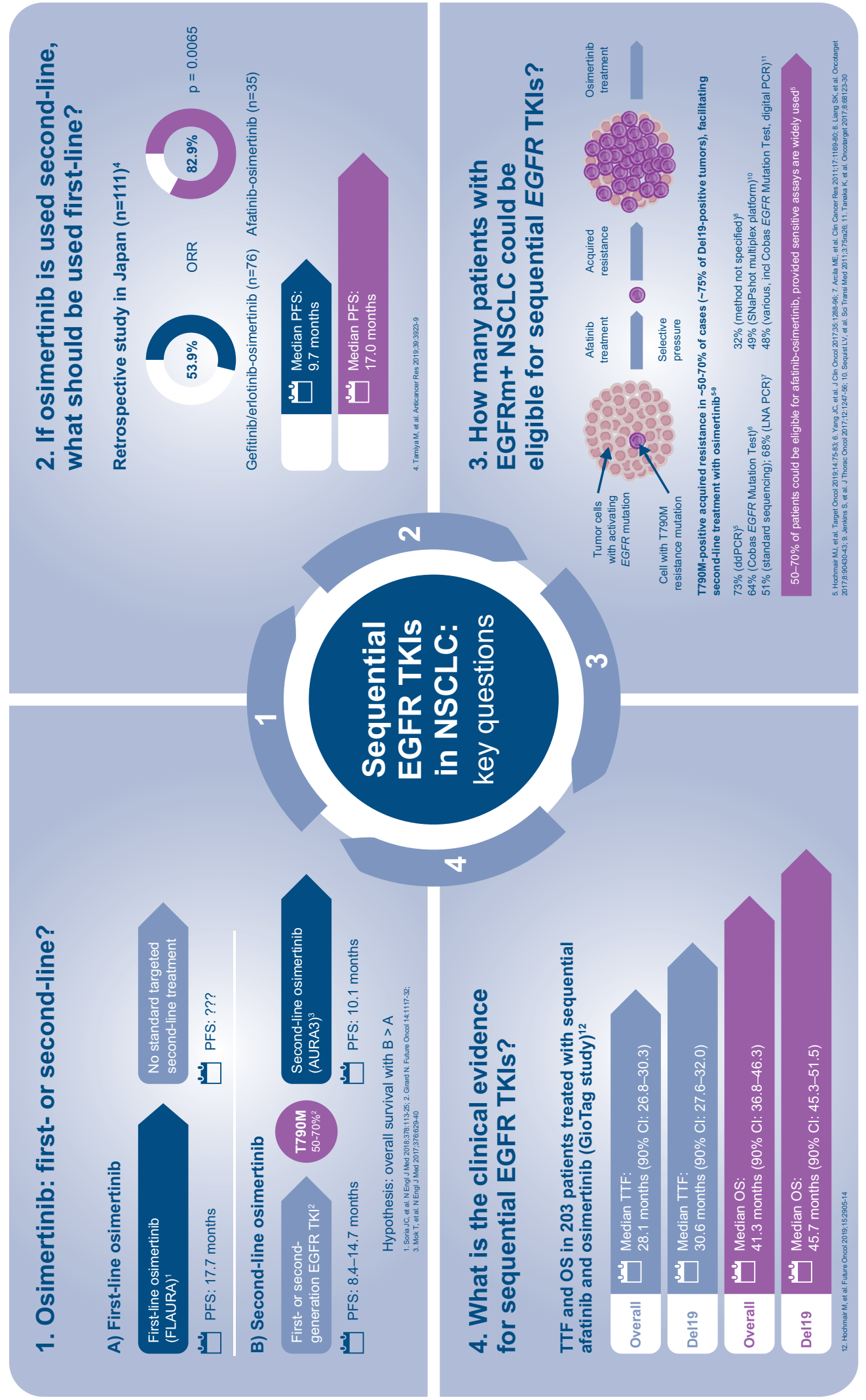